Journal of The Korean Astronomical Society

41: $139 \sim 145,2008$ October

(C)2008 The Korean Astronomical Society. All Rights Reserved.

\title{
MOLECULAR CLOUD ASSOCIATED WITH AFGL 2591
}

\author{
Y. C. MINH ${ }^{1}$ AND JI YANG ${ }^{2}$ \\ ${ }^{1}$ Korea Astronomy and Space Science Institute, 838 Daeduk-daero, Yuseong-gu, Daejeon 305-348, Korea E-mail: \\ minh@kasi.re.kr \\ 2 Purple Mountain Observatory, Chinese Academy of Sciences, 2 Beijing Xi Lu, Nanjing, Jiangsu 210008, China \\ E-mail: jiyang@pmo.ac.cn \\ (Received September 24, 2008; Accepted October 6, 2008)
}

\begin{abstract}
The molecular cloud, embedding AFGL 2591, has a "head-and-tail" structure with a total mass of $\sim 1800 \mathrm{M}_{\odot}$, about half of the mass $\left(\sim 900 \mathrm{M}_{\odot}\right)$ in the head (size $\sim 1.2 \mathrm{pc}$ in diameter), and another half in the envelope $(\sim 3.5 \mathrm{pc}$ in the east-west direction). We found a new cloud in the direction toward north-east from AFGL 2591 (projected distance $\sim 2.4 \mathrm{pc}$ ), which is probably associated with the AFGL 2591 cloud. The ${ }^{12} \mathrm{CO}$ spectrum clearly shows a blue-shifted high-velocity wing at around the velocity $-20 \sim-10 \mathrm{~km} \mathrm{~s}^{-1}$, but it is not clear whether this high-velocity component has a bipolar nature in our observations. The observed CN spectra also show blue-shifted wing component but the existence of the red-shifted component is not clear, either. In some CN and HCN spectra, the highvelocity components appear as a different velocity component, not a broad line-wing component. The dense cores, traced by CN and HCN, exist in the 'head' of the AFGL 2591 cloud with an elongated morphology roughly in the north-south direction with a size of about $0.5 \mathrm{pc}$. The abundance ratio between $\mathrm{CN}$ and $\mathrm{HCN}$ is found to be about $2-3$ within the observed region, which may suggest a possibility that this core is being affected by the embedded YSOs or by possible shocks from outside.
\end{abstract}

Key words : ISM: abundances - ISM: individual - AFGL 2591 - stars: formation

\section{INTRODUCTION}

AFGL 2951 is a massive young stellar object (YSO) embedded in a dense molecular cloud in the Cygnus-X region, which was first recognized as a molecular outflow source by Bally \& Lada (1983). The distance to AFGL 2591 is assumed $1 \mathrm{kpc}$, but very uncertain because of the high galactic longitude $\left(\sim 79^{\circ}\right)$ and obscuration by massive dust shell (van der Tak et al., 1999; Trinidad et al., 2003). At this distance, it has a luminosity of $\sim 2 \times 10^{4} \mathrm{~L}_{\odot}$ and an estimated stellar mass of $10 \mathrm{M}_{\odot}$, which is presently observed in the process of breaking out of its natal cocoon (cf. Lada et al., 1984; van der Tak et al., 1999). This source shows energetic star formation activities, and several compact radio sources were found within the region, which has led to the suggestion that multiple star formations have occurred (Campbell, 1984). This source is known as a powerful molecular outflow source, which is extended more than $5^{\prime} \times 5^{\prime}$ with a dynamical age of $2 \times 10^{4}$ yrs (Bally \& Lada, 1983; Lada et al., 1984).

AFGL 2591 has been a target for extensive studies on the circumstellar envelop because of its relatively isolated existence, but most studies have been concentrated to disclose the nature of the embedded source itself and its very surrounding within roughly about $1^{\prime}$ angular diameter region (e.g., van der Tak et al., 1999;

Corresponding Author: Y. C. Minh
Preibisch et al., 2003; Hutawarakorn \& Cohen, 2005, and the references therein). Our aim is to study on the molecular clouds embedding AFGL 2591, since, after the onset of massive star formation, the various energetic activities associated with the YSOs should have altered the surroundings in a relatively short time scale. For example, energetic large-scale outflows of molecular gas near YSOs represent extremely important phase of early stellar evolution common to stars of a wide range of spectral type. The kinetic energies of outflows, in general, are enormous (i.e., $10^{45}-10^{47} \mathrm{ergs}$ ) and may represent a significant input of mechanical energy into molecular clouds, which may modify their surroundings significantly (Lada et al., 1984).

In this paper, we study the larger scale, compared to the embedded source scale, gas structure and properties around AFGL 2591, using the $3 \mathrm{~mm}$ transition observations of $\mathrm{CO}, \mathrm{HCN}$, and $\mathrm{CN}$. In $\S$ II we summarize the observations, the results and discussion are included in $\S$ III, and the summary in $\S$ IV.

\section{OBSERVATIONS}

Observations were carried out using the $13.7 \mathrm{~m}$ millimeter-wave radio telescope of Purple Mountain Observatory at Delingha, China at April 2006. We observed the ${ }^{12} \mathrm{CO}$ and ${ }^{13} \mathrm{CO} \mathrm{J}=1-0$ transition lines using the multi-line backend (Zuo et al., 2004). An SIS receiver in double sideband (DSB) mode was used as 


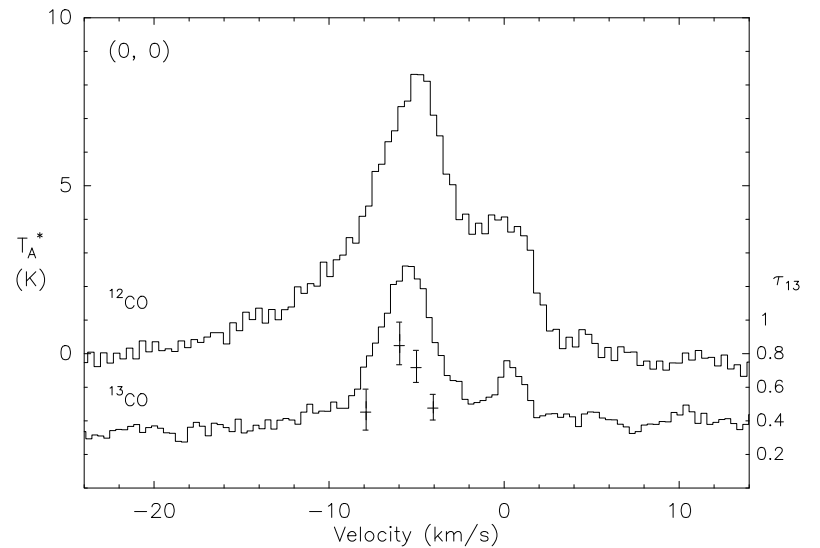

Fig. 1.- Sample spectra of the observed $1-0$ lines of ${ }^{12} \mathrm{CO}$ and ${ }^{13} \mathrm{CO}$ obtained toward the offset $(0,0)$ position

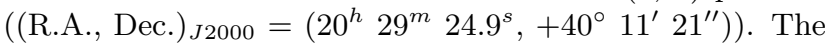
optical depths of the ${ }^{13} \mathrm{CO} 1-0$ line are indicated in several velocities and the optical depth scale is shown in the right side of the box.

the front-end and the backend system contains three acousto-optical spectrometers of 1024 channels, with a bandwidth of $145 \mathrm{MHz}$ for the ${ }^{12} \mathrm{CO}$ and $43 \mathrm{MHz}$ for the ${ }^{13} \mathrm{CO}$ lines. The CN $1-0 J=3 / 2-1 / 2$ hyperfine lines were observed using the same front end and one of the narrow-band AOS spectrometers.

We observed the ${ }^{12} \mathrm{CO}$ and ${ }^{13} \mathrm{CO} \mathrm{J}=1-0$ transitions, the $\mathrm{HCN} 1-0$ hyperfine lines, and $\mathrm{CN} 1-0 \mathrm{~J}=$ $3 / 2-1 / 2$ hyperfine lines at 3 millimeters. The positionswitching mode was used with an on-source integration time of 1 minute for each position. The data were reduced with NEWSTAR of NROJ (National Radio Observatory of Japan) and the GILDAS software package from IRAM (Institut de Radioastronomie Millimétrique). The antenna temperature, $\mathrm{T}_{\mathrm{A}}^{*}$, quoted in this paper has been corrected for antenna and atmospheric losses by means of the standard chopper wheel method, but not for possible beam dilution. The HPBW and main beam efficiency at zenith are $55^{\prime \prime}$ and 0.66 , respectively, at $100 \mathrm{GHz}$, and $48^{\prime \prime}$ and 0.63 , respectively, at $115 \mathrm{GHz}$. Elevation dependence of antenna efficiencies has been corrected by using nearby standard sources. The typical system temperatures $\left(\mathrm{T}_{\mathrm{DSB}}\right)$ were about $230-260 \mathrm{~K}$ for $\mathrm{CO}, \sim 210 \mathrm{~K}$ for $\mathrm{CN}$, and $\sim 300 \mathrm{~K}$ for HCN observations. And the typical RMS $(1 \sigma)$ values of the spectra are $0.20-0.25 \mathrm{~K}$ for $\mathrm{CO}$ data and $0.04-0.06 \mathrm{~K}$ for $\mathrm{CN}$ and $\mathrm{HCN}$ data. With the AFGL 2591 position as the map center, (R.A., Dec. $)_{J 2000}=\left(20^{h} 29^{m} 24.9^{s},+40^{\circ} 11^{\prime} 21^{\prime \prime}\right)$, CO maps were made with $1^{\prime}$ spacings and $\mathrm{HCN}$ and $\mathrm{CN}$ maps with $0.5^{\prime}$ spacings. The observed positions are indicated in the figures.

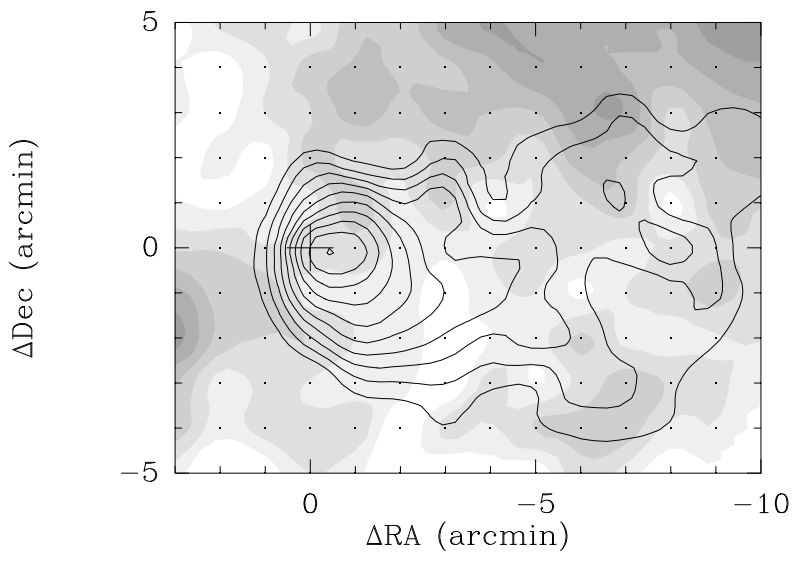

Fig. 2. - Integrated intensity map of the ${ }^{13} \mathrm{CO} 1-0$ line for the velocity range $v=-11 \sim-3 \mathrm{~km} \mathrm{~s}^{-1}$ (contour lines) and $v=-3 \sim+3 \mathrm{~km} \mathrm{~s}^{-1}$ (grey scale). The contour lines start from $5 \mathrm{~K} \mathrm{~km} \mathrm{~s}^{-1}$ and increase by $1.5 \mathrm{~K} \mathrm{~km} \mathrm{~s}^{-1}$. The grey scale increases with a $1 \mathrm{~K} \mathrm{~km} \mathrm{~s}^{-1}$ step from the lowest $3 \mathrm{~K} \mathrm{~km} \mathrm{~s}^{-1}$. The dots are observed points, and the cross at the $(0,0)$ position indicates the AFGL 2591 position.

\section{RESULTS AND DISCUSSION}

\section{(a) Morphology of the Cloud Embedding AFGL 2591}

Fig. 1 shows sample spectra obtained toward the $(0$, $0)$ position, which clearly shows two velocity components peaked at $v_{l s r} \approx-5.6$ and $0.3 \mathrm{~km} \mathrm{~s}^{-1}$ (from the gaussian fit of the ${ }^{13} \mathrm{CO}$ spectrum). The $v_{l s r} \approx-5.7$ $\mathrm{km} \mathrm{s}^{-1}$ is known as the systemic velocity of the molecular cloud embedding AFGL 2591 (e.g., van der Tak et al., 1999). The velocity integrated intensity map for these two component is shown in Fig. 2. The -5.7 $\mathrm{km} \mathrm{s}^{-1}$ component is concentrated around AFGL 2591 with an envelope extended toward the west direction. This morphology shows a "head-and-tail" structure, extended by more than $3.5 \mathrm{pc}$ (at $1 \mathrm{kpc}$ distance) along the east-west direction. But the dense core ("head") containing AFGL 2591 shows roughly a circular shape with a mean diameter of about $1.2 \mathrm{pc}(\mathrm{HPW})$. The near-infrared image also shows a similar core-envelope structure in Fig. 2 (Burns et al., 1989). We don't see a significant velocity gradient along the ease-west direction, which will be further discussed in $\S(\mathrm{b})$. On the other hand, the component at $v_{l s r} \sim 0.3 \mathrm{~km} \mathrm{~s}^{-1}$ (the $0 \mathrm{~km} \mathrm{~s}^{-1}$ component, hereafter) appears to be extended in a larger area than the observed region, as shown with grey scale in Fig. 2. This component needs to be studied further to see whether it is a background/foreground component or directly associated with the molecular cloud containing AFGL 2591.

Optical depths of the ${ }^{13} \mathrm{CO} 1-0$ line of the $(0,0)$ position were estimated to be less than 0.8 in several velocities, and included in Fig. 1. These optical depths $\left(\tau_{13}\right)$ were derived by comparing the line intensities with that of ${ }^{12} \mathrm{CO}$, assuming the same rotational tem- 


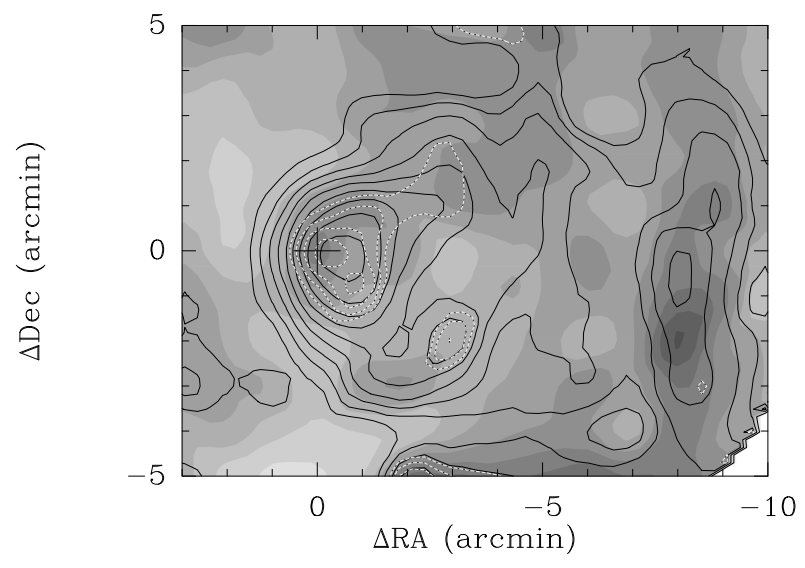

Fig. 3. - Integrated intensity map of the ${ }^{12} \mathrm{CO} 1-0$ line for the velocity range $v=-20 \sim-3 \mathrm{~km} \mathrm{~s}^{-1}$ (contour lines) and $v=-3 \sim+3 \mathrm{~km} \mathrm{~s}^{-1}$ (grey scale). The dotted lines show the high-velocity component integrated in the velocity range $v=-20 \sim-10 \mathrm{~km} \mathrm{~s}^{-1}$. The contour lines start from $10 \mathrm{~K} \mathrm{~km} \mathrm{~s}^{-1}$ and increase by $5 \mathrm{~K} \mathrm{~km} \mathrm{~s}^{-1}$. The grey scale increases with a $2 \mathrm{~K} \mathrm{~km} \mathrm{~s}^{-1}$ step from the lowest $5 \mathrm{~K} \mathrm{~km}$ $\mathrm{s}^{-1}$. The dotted lines increases with a $1.5 \mathrm{~K} \mathrm{~km} \mathrm{~s}^{-1}$ step from the lowest $5 \mathrm{~K} \mathrm{~km} \mathrm{~s}^{-1}$.

peratures and the abundance ratio $\sim 45$ between ${ }^{12} \mathrm{CO}$ and ${ }^{13} \mathrm{CO}$. For the total column density derivation, we assume optically thin emission and an LTE condition, and derive $\mathrm{N}\left({ }^{13} \mathrm{CO}\right)=2.9 \pm 0.5 \times 10^{16} \mathrm{~cm}^{-2}$ toward the peak position. The errors come from the applied rotational temperature range, $10-20 \mathrm{~K}$. Observed spectral noise $(1 \sigma)$ gives $\lesssim 5 \%$ further uncertainty. Then we derive the mass of the "head" $\sim 8.2 \pm 1.6 \times 10^{2} \mathrm{M}_{\odot}$, using the source size, $\sim 1.2 \mathrm{pc}$ in diameter, and applying the $\mathrm{N}\left({ }^{13} \mathrm{CO}\right) / \mathrm{N}\left(\mathrm{H}_{2}\right)$ conversion ratio suggested by Harjunpää, Lehtinen, \& Haikala $(2004)\left(\sim 8 \times 10^{-7}\right.$, by averaging the slope values estimated for three globules, without the offset). On the other hand, by integrating the all observed points within the lowest contour line ( $\sim 5 \sigma$ noise level) in Fig. 2, the total mass of the "headand-tail" cloud is estimated to be about $1.8 \pm 0.4 \times 10^{3}$ $\mathrm{M}_{\odot}$. Therefore about half of the mass is concentrated in the AFGL 2591 core ("head") and another half in the envelope.

In Fig. 3 we show the ${ }^{12} \mathrm{CO} 1-0$ intensity map, which also shows similar morphology as that of ${ }^{13} \mathrm{CO}$ with a little more complexity. The ${ }^{12} \mathrm{CO}$ spectrum (Fig. 1) clearly shows a blue-shifted high-velocity wing at around the velocity $-20 \sim-10 \mathrm{~km} \mathrm{~s}^{-1}$, and its velocity integrated intensity map is also included in Fig. 3 as dotted-line contours. This high-velocity component appears to be mainly confined to AFGL 2591 and extended toward west. AFGL 2591 is already known to have a significant outflow component in infrared and radio observations toward the embedded source direction (e.g., Mitchell et al., 1989; van der Tak et al., 1999). It is not clear, however, whether this high-velocity component has a bipolar nature in our observations, since

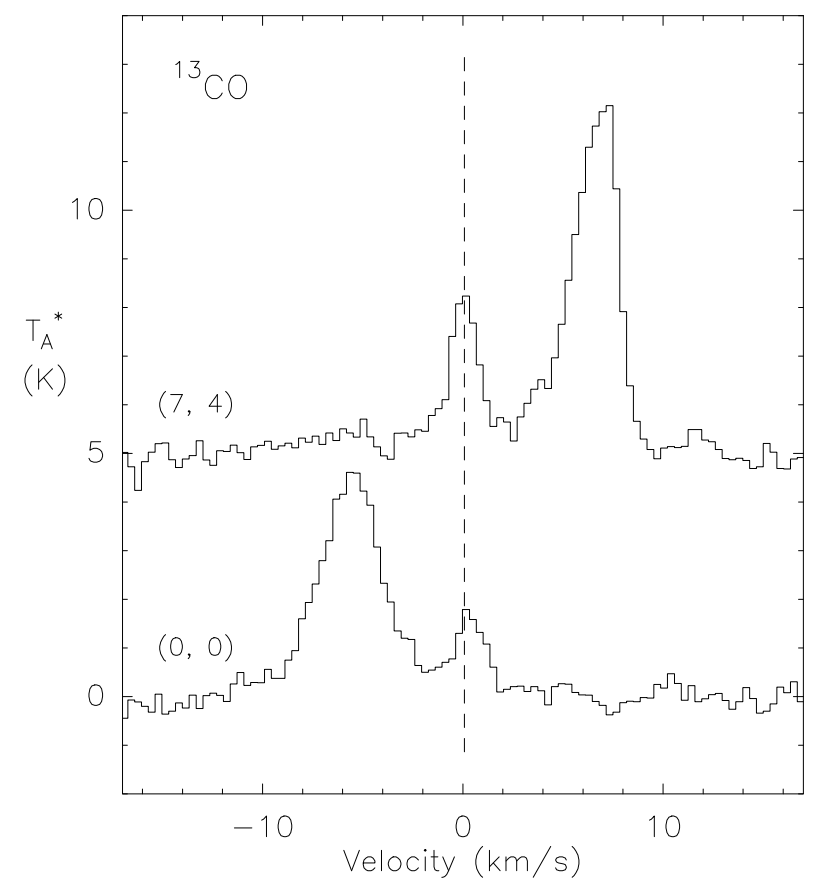

Fig. 4.- ${ }^{13} \mathrm{CO} 1-0$ lines obtained toward the offsets $(0$, $0)$ and $\left(7^{\prime}, 4^{\prime}\right)$ position. The dashed line simply shows the position of the $0 \mathrm{~km} \mathrm{~s}^{-1}$.

the red-shifted component, if there is any, appears to be overlapped with the $0 \mathrm{~km} \mathrm{~s}^{-1}$ component. But this source does not show a large-scale outflow (broad wing components extended more than a few arcminutes) in our observations, which need to be studied further with more sensitive observations.

\section{(b) A New Molecular Cloud Associated with the AFGL 2591 Cloud}

One of our aims is to investigate the cloud structure in a larger scale than the cloud embedding AFGL 2591, which may have caused the initial collapse of the massive star formation, including the AFG 2591 cloud and its surroundings. The molecular cloud embedding AFGL 2591 has a well bounded shape having a core and an extended envelope as a "head-and-tail" structure, but we also found the ${ }^{13} \mathrm{CO} 1-0$ line emission toward the north-east direction from AFG L2591. Fig. 4 shows spectra taken at the $\left(7^{\prime}, 4^{\prime}\right)$ offset position from AFGL 2591, together with the $(0,0)$ spectrum. And this figure shows three velocity components, at around $-5.7,0,+7 \mathrm{~km} \mathrm{~s}^{-1}$, which are distinguishable clearly. Fig. 5 shows the distributions of these three different velocity components. The $-5.7 \mathrm{~km} \mathrm{~s}^{-1}$ component is shown with dotted contours, which is the same map in Fig. 2. The $0 \mathrm{~km} \mathrm{~s}^{-1}$ component is extended further than the observed region and need to be studied further as mentioned in the previous section. The $7 \mathrm{~km}$ $\mathrm{s}^{-1}$ cloud (the NE cloud) also shows a core and envelope structure similar to the $-5.7 \mathrm{~km} \mathrm{~s}^{-1}$ cloud (the 


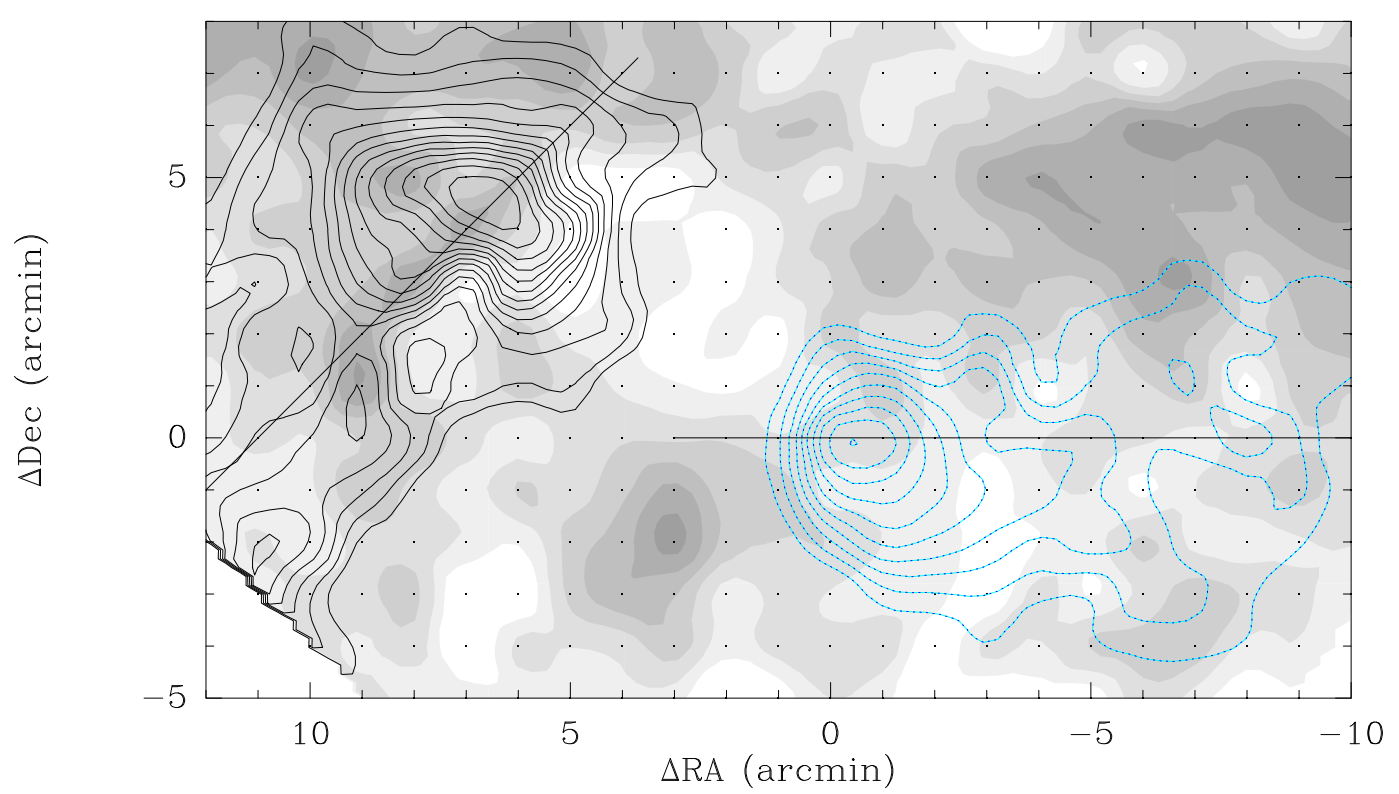

Fig. 5. - Integrated intensity map of the ${ }^{13} \mathrm{CO} 1-0$ line for the velocity range $v=-11 \sim-3 \mathrm{~km} \mathrm{~s}^{-1}$ (dotted contour lines, as shown in Fig. 2), $v=+3 \sim+11 \mathrm{~km} \mathrm{~s}^{-1}$ (solid contour lines), and the $0 \mathrm{~km} \mathrm{~s}^{-1}$ component (grey scale for $v=-3 \sim+3$ $\mathrm{km} \mathrm{s}^{-1}$ ). Both contour levels start from $5 \mathrm{~K} \mathrm{~km} \mathrm{~s}^{-1}$ and increase by $1.5 \mathrm{~K} \mathrm{~km} \mathrm{~s}^{-1}$. The grey scale increases with a $1 \mathrm{~K} \mathrm{~km}$ $\mathrm{s}^{-1}$ step from the lowest $3 \mathrm{~K} \mathrm{~km} \mathrm{~s}^{-1}$. The dotted lines around the $(0,0)$ position is the same map in Fig. 2. The two solid straight lines show the positions to derive the position-velocity maps in Fig. 6 .

AFGL 2591 cloud). But the total mass of the NE cloud is larger by $30 \%$ for the core $\left(\sim 10^{3} \mathrm{M}_{\odot}\right)$ and $45 \%$ for the total mass $\left(\sim 2.6 \times 10^{3} \mathrm{M}_{\odot}\right)$ than the AFGL 2591 cloud. The central point between two clouds is at about the $\left(3.5^{\prime}, 2.2^{\prime}\right)$ offset position from AFGL 2591, then the projected distance from the center to the sources is about $1.2 \mathrm{pc}$ assuming a $1 \mathrm{kpc}$ distance to the source.

Fig. 6 shows the position-velocity map for both clouds along the line indicated in Fig. 5. We did not find, however, a noticeable velocity gradient $(\lesssim 0.3 \mathrm{~km}$ $\mathrm{s}^{-1} \mathrm{pc}^{-1}$ ) along the long axis from the head to the tail. Therefore it is not clear whether these two clouds are a binary system, but they seem to be associated with each other, and possible interactions between two clouds may have influenced the star formation in the AFGL 2591 core.

\section{(c) $\mathrm{CN}$ and $\mathrm{HCN}$}

Fig. 7 shows the spectrum of $\mathrm{CN}$ and $\mathrm{HCN}$ obtained toward the $(0,0)$ position. The $\mathrm{CN}$ map in Fig. 8 was derived for the $F=5 / 2-3 / 2$ component only, but all 3 hyperfine components of $\mathrm{HCN}$ were integrated together because of the difficulty to distinguish. The map shows that both these emissions do not peak toward AFGL 2591, but toward $\sim 0.5^{\prime}$ east from AFGL 2591. The molecular clump, traced by $\mathrm{CN}$ and HCN, is concentrated in the 'leading' part of the 'head' of this molecular cloud, and elongated roughly in the northsouth direction, with a mean diameter of about $0.5 \mathrm{pc}$ (at $1 \mathrm{kpc}$ distance). There exist line-wing components of the observed $\mathrm{CN}$ and HCN lines, as shown in Fig. 7, roughly at $\sim-8 \mathrm{~km} \mathrm{~s}^{-1}$. We show integrated intensity maps of this component in Fig. 8, with black solid contour lines. In the observed spectra the red-shifted component does not seem to exist. We also made a same map for HCN and included in the figure. In general the high-velocity component shows similar morphology with the main $\left(-5.7 \mathrm{~km} \mathrm{~s}^{-1}\right)$ component, elongated roughly in the north-south direction. However, as you see in the HCN spectrum in Fig. 7, the "line-wing" component at around $-8 \mathrm{~km} \mathrm{~s}^{-1}$ looks like a different velocity component instead of line-wing component. In other positions the CN spectra also show a separate component at this velocity instead of the line-wing feature. Thus we think that this component may simply a different gas component having a slightly different velocity. This needs to be studied further, again with higher and more sensitive observations.

We derive the beam-averaged total column density of $\mathrm{CN}$ and $\mathrm{HCN}, 1.4 \pm 0.13 \times 10^{13} \mathrm{~cm}^{-2}$ and $3.8 \pm 0.10 \times$ $10^{13} \mathrm{~cm}^{-2}$, respectively, at the peak position, by assuming optically thin emission and an LTE condition. The errors come from the applied rotational temperature range, $5-10 \mathrm{~K}$, and the observed spectral noise $(1 \sigma)$ gives an further uncertainty $\lesssim 10 \%$. The abundance ratio between $\mathrm{CN}$ and $\mathrm{HCN}, \mathrm{N}(\mathrm{CN}) / \mathrm{N}(\mathrm{HCN})$, is about $2-3$ within the observed region. $\mathrm{HCN}$ is commonly used as a tracer of dense molecular gas (with densities $\mathrm{n}_{\mathrm{H}_{2}} \gtrsim 10^{4} \mathrm{~cm}^{-3}$ ), and CN, which is chemically linked to $\mathrm{HCN}$, is also another tracer of dense gas, with a lower (by a factor of 5) critical density than HCN (e.g., 

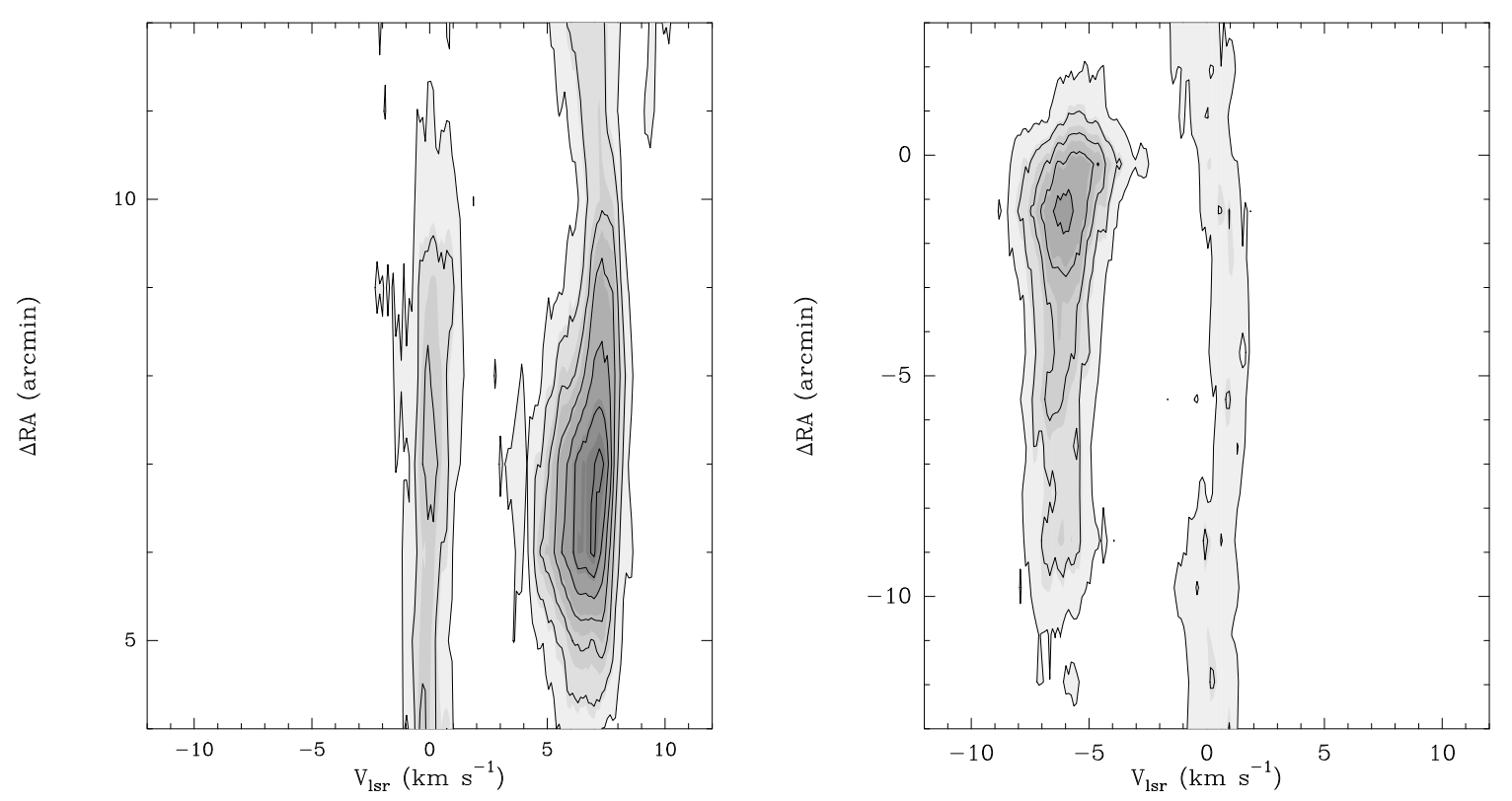

Fig. 6. - Position-velocity maps of the ${ }^{13} \mathrm{CO} 1-0$ line obtained (in $\mathrm{T}_{\mathrm{A}}^{*}$ scale) (left) along the offsets between $\left(7^{\prime}, 4^{\prime}\right)$ and $\left(12^{\prime},-1^{\prime}\right)$, and $($ right $)$ along the offsets between $\left(3^{\prime}, 0^{\prime}\right)$ and $\left(-10^{\prime}, 0^{\prime}\right)$ as indicated with straight lines in Fig. 5 . Both contour lines increase by a $1 \mathrm{~K}$ step from the lowest $1 \mathrm{~K}$.

Pérez-Beaupuits, Aalto, \& Gerebro, 2007). In the quiet molecular region the $\mathrm{N}(\mathrm{CN}) / \mathrm{N}(\mathrm{HCN})$ ratio is expected to be $\sim 0.01-0.1$. Both $\mathrm{CN}$ and $\mathrm{HCN}$ are also known to be especially abundant in PDRs (photodissociation regions), and the $\mathrm{N}(\mathrm{CN}) / \mathrm{N}(\mathrm{HCN}) \geq 1$ has been suggested as a diagnostic tool to trace a PDR, since the $\mathrm{CN}$ abundance can further be enhanced by the direct photodissociation of HCN (cf., Thi, van Zadelhoff, \& van Dishoeck, 2004, and references therein). Since there are many uncertainties in derivation of $\mathrm{CN}$ and $\mathrm{HCN}$ abundances from these one transition observations, it is difficult to discuss details on the abundance ratio in this source. Therefore we only suggest that the ratio, $\mathrm{N}(\mathrm{CN}) / \mathrm{N}(\mathrm{HCN}) \sim 2-3$ in this $\mathrm{CN} / \mathrm{HCN}$ core, may result from either by the interaction with outflows from AFGL 2591, as suggested by the $\mathrm{H}_{2}$ emission feature (Tamura \& Yamashita, 1992), or by shocks from the outside, as suggested by the elongated morphology of $\mathrm{CN}$ and $\mathrm{HCN}$ emitting regions.

\section{SUMMARY}

The molecular cloud, embedding a massive YSO AFGL 2591, has a core and envelope morphology of a "head-and-tail" structure. We derive a total mass of $\sim 1800 \mathrm{M}_{\odot}$, about half of the mass $\left(\sim 900 \mathrm{M}_{\odot}\right)$ in the "head" with a size of about $\sim 1.2 \mathrm{pc}$ in diameter, and another half in the envelope elongated toward the west direction with $\gtrsim 3.5 \mathrm{pc}$ in size, assuming a $1 \mathrm{kpc}$ distance.

The ${ }^{12} \mathrm{CO}$ spectrum clearly shows a blue-shifted high-velocity wing at around the velocity $-20 \sim-10$ $\mathrm{km} \mathrm{s}^{-1}$. This component is mainly confined to the
AFGL 2591 core and extended toward west with a size of about $2^{\prime}$. It is not clear, however, whether this highvelocity component has a bipolar nature in our observations. The observed CN spectra also show blue-shifted wing component but the existence of the red-shifted component is also not clear. The nature of the broad wing component needs to be studied further.

We found a new cloud component (the NE cloud) at a distance of about $2.4 \mathrm{pc}$ toward north-east direction from AFGL 2591, which also has a core and envelope structure, like the AFGL 2591 cloud. We did not find, however, a noticeable velocity gradient $\left(\lesssim 0.3 \mathrm{~km} \mathrm{~s}^{-1}\right.$ $\mathrm{pc}^{-1}$ ) along the long axis of the clouds, and it is not clear whether these two clouds are a binary system.

The dense cores, traced by $\mathrm{CN}$ and $\mathrm{HCN}$, is found to be located in the 'head' of the AFGL 2591 cloud toward the 'leading' direction of this molecular cloud. The morphology of this core is elongated roughly in the north-south direction with a size of about $0.5 \mathrm{pc}$. The abundance ratio between $\mathrm{CN}$ and $\mathrm{HCN}$ is found to be about $2-3$ within the observed region, which may suggest that this core is being affected by the embedded YSOs or by shocks from the outside.

\section{ACKNOWLEDGEMENTS}

This work was supported by the Korea Science and Engineering Foundation(KOSEF) grant funded by the Korea government(MEST) (No. R01-2008-000-200020(2008)). 


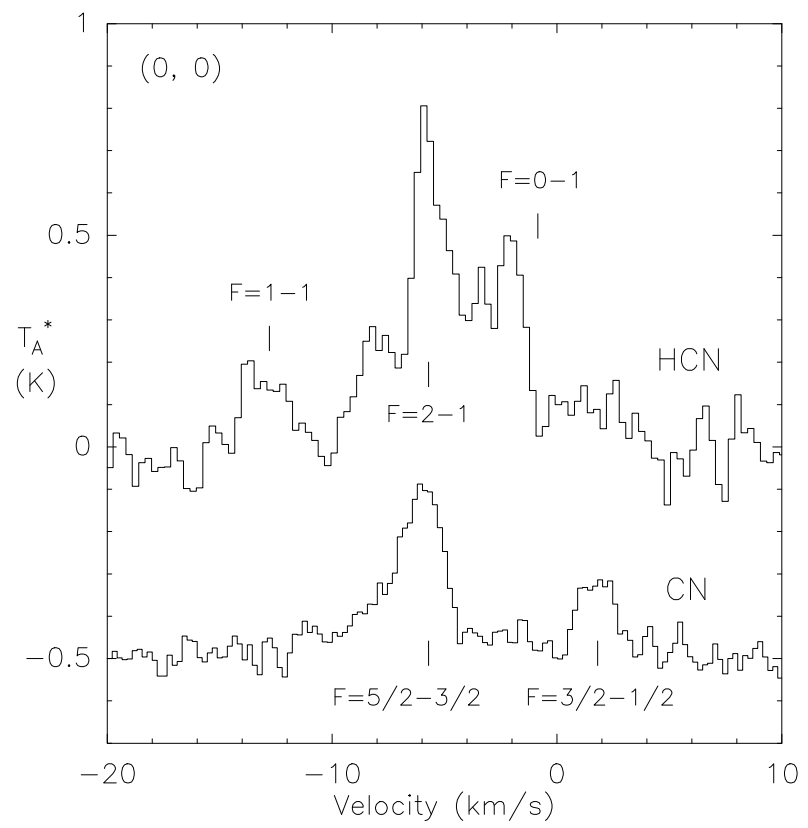

Fig. 7.- Observed HCN $1-0$ and CN $1-0 \mathrm{~J}=3 / 2-1 / 2$ lines toward the $(0,0)$ position. The observed hyperfine components are indicated.

\section{REFERENCES}

Bally, J. \& Lada, C. J., 1983, The high-velocity molecular flows near young stellar objects, ApJ, 265, 824

Burns, M. S., Hayward, T. L., Thronson, Jr., H. A., \& Johnson, P. E., 1989, Imaging polarimetry of AFGL 2591 and its associated reflection nebula, AJ, 98, 659

Campbell, B., 1984, New radio sources at AFGL 2591 - Young cluster or single star?, ApJ, 287, 334

Carr, J. S., Evans II, N. J., Lacy, J. H., \& Zhou, S., 1995, Observation of Infrared and Radio Lines of Molecules toward GL 2591 and Comparison to Physical and Chemical Models, ApJ, 450, 667

Harjunpää, P., Lehtinen, K., \& Haikala, L. K., 2004, The relationship of $\mathrm{CO}$ abundance to extinction and $\mathrm{N}(\mathrm{H} 2)$ :. Observations of globules and the dependence on star formation activity, A\&A, 421, 1087

Hutawarakorn, B. \& Cohen, R. J., 2005, OH maser disc and magnetic field structure in AFGL 2591, MNRAS, 357, 338

Lada, C. J., Thronson Jr., H. A., Smith, H. A., Schwartz, P. R., \& Glacuum, W., 1984, The nature of AFGL 2591 and its associated molecular outflow Infrared and millimeter-wave observations, ApJ, 286, 302

Mitchell, G. F., Curry, C., Maillard, J.-P., \& Allen, M., 1989, The gas environment of the young stellar object GL 2591 studied by infrared spectroscopy, ApJ, 341, 1020
Pérez-Beaupuits, J. P., Aalto, S., \& Gerebro, H., 2007, HNC, HCN and CN in Seyfert galaxies, A\&A, 476, 177

Preibisch, T., Balega, Y. Y., Schertl, D., \& Weigelt, G., 2003, Hiding the high excitation in the head of a fast Herbig-Haro jet, A\&A, 412, 735

Tamura, M. \& Yamashita, T., 1992, Infrared morphology of mass outflow from GL 2591, ApJ, 391, 710

Thi, W.-F., van Zadelhoff, G.-J., \& E. F. van Dishoeck, E. F., 2004, Organic molecules in protoplanetary disks around $\mathrm{T}$ Tauri and Herbig Ae stars, A\&A, 425,955

Trinidad, M. A., Curiel, S., Cantó, J., D’Alsssio, P., Rodríguez, L. F., Torrelles, J. M., Goómez, J. F., Patel, N., \& Ho, P. T. P., 2003, Observations of Water Masers and Radio Continuum Emission in AFGL 2591, ApJ, 589, 386

van der Tak, F. F. S., van Dishoeck, E. F., Evans II, N. J., Bakker, E. J., \& Blake, G. A., 1999, The Impact of the Massive Young Star GL 2591 on Its Circumstellar Material: Temperature, Density, and Velocity Structure, ApJ, 522, 991

Zuo, Y.-X., Yang, J., Shi, S.-C., Chen, S.-H., Pei, L.B., Yao, Q.-J., Sun, J.-J. \& Lin, Z.-H., 2004, Upgrade Procedure for the Delingha 13.7-m Telescope, ChJAA, 4, 390 


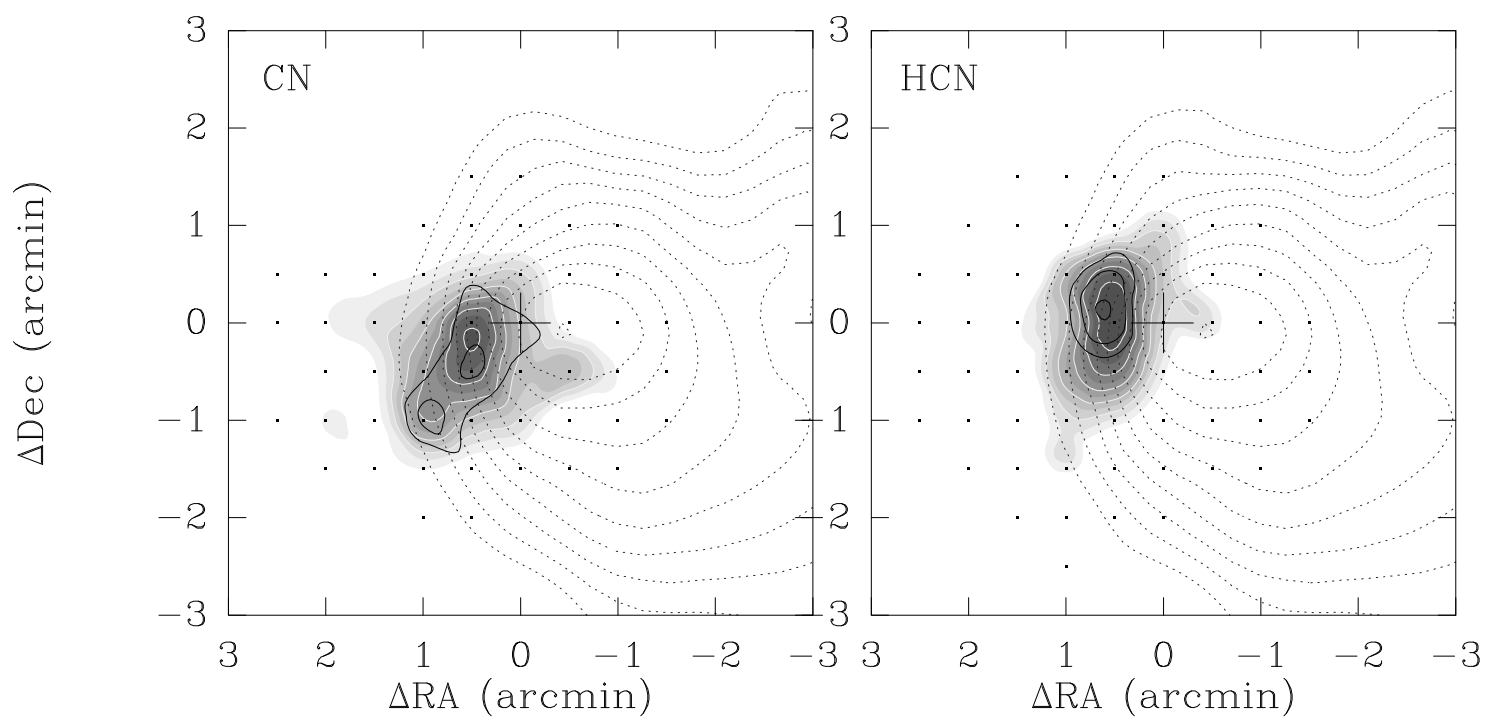

Fig. 8.- (Left) Integrated intensity map of the observed $\mathrm{CN}$ and $\mathrm{HCN}$ lines. The CN $1-0$ lines were integrated in the velocity range $v=-7.5 \sim-3 \mathrm{~km} \mathrm{~s}^{-1}$ (grey scale and white contours) for one hyperfine component $(1-0 \mathrm{~J}=3 / 2-1 / 2 \mathrm{~F}=$ $5 / 2-3 / 2$ ) shown in Fig. 7 , and for the high-velocity component $v=-11 \sim-7.5 \mathrm{~km} \mathrm{~s}^{-1}$ (black contours). The white contour lines increase by a $0.2 \mathrm{~K} \mathrm{~km} \mathrm{~s}^{-1}$ and the highest contour level is $1.6 \mathrm{~K} \mathrm{~km} \mathrm{~s}^{-1}$. The black contour lines increase from 0.3 $\mathrm{K} \mathrm{km} \mathrm{s}^{-1}$ by $0.1 \mathrm{~K} \mathrm{~km} \mathrm{~s}^{-1}$. The observed positions are indicated as dots and the cross indicates the AFGL 2591 position. The dotted contours are the integrated intensity map of ${ }^{13} \mathrm{CO}$ in Fig. 2. (Right) The HCN $1-0$ lines were integrated in the velocity range $v=-20 \sim+5 \mathrm{~km} \mathrm{~s}^{-1}$ (grey scale and white contours) to include 3 hyperfine lines shown in Fig. 7, and for the high-velocity component $v=-11 \sim-7.5 \mathrm{~km} \mathrm{~s}^{-1}$ (black contours). The white contours increase by a $0.6 \mathrm{~K} \mathrm{~km} \mathrm{~s}^{-1}$ and the highest contour level is $6 \mathrm{~K} \mathrm{~km} \mathrm{~s}^{-1}$. The black contours increase from $0.8 \mathrm{~K} \mathrm{~km} \mathrm{~s}^{-1}$ by $0.1 \mathrm{~K} \mathrm{~km} \mathrm{~s}^{-1}$. 